\title{
Autonomous mesoscale positioning emerging from spatiotemporally controlled coupling between self-assembly and gradient-driven molecular fluxes
}

Arno van der Weijden, Mitch Winkens, Sandra M. C. Schoenmakers, Wilhelm T. S. Huck \& Peter A. Korevaar*

Institute for Molecules and Materials, Radboud University, Nijmegen, The Netherlands.

*e-mail:p.korevaar@science.ru.nl

Out-of-equilibrium molecular systems hold great promise as dynamic, reconfigurable matter that executes complex tasks autonomously. However, translating molecular scale dynamics into spatiotemporally controlled phenomena at mesoscopic length scales remains a challenge. In living cells, reliable positioning processes such as the centering of the centrosome involve forces that result from dissipative self-assembly. We demonstrate how spatiotemporal positioning emerges in synthetic systems where self-assembly is coupled to molecular fluxes originating from concentration gradients. At the core of our system are millimeter long self-assembled filaments and Marangoni flows induced by non-uniform amphiphile distributions. We demonstrate how repulsive and attractive forces that are generated as filaments organize between source and drain droplets sustain autonomous positioning of dynamic assemblies at the mesoscale. Our concepts provide a new paradigm for the development of non-equilibrium matter with spatiotemporal programmability.

Living matter is driven by well-regulated systems of chemical reactions that coordinate selfassembly, process information and transmit signals to organize cellular components in time and space. ${ }^{1}$ Functions that emerge from these systems offer great inspiration for the design of self-organizing systems that display behavior such as motion ${ }^{2-5}$, pattern formation ${ }^{6}$ or complex shape transformations. ${ }^{7-10}$ Whereas the molecular assembly of static, equilibrium structures is now well-understood, it is clear that the development of functional matter with life-like features requires a paradigm where molecules are guided along their assembly pathways under out-of-equilibrium conditions. ${ }^{11-18}$ In nature, for example, the construction of the cellular cytoskeleton shows how coupling of molecular assembly to dissipative chemical reactions leads to self-organization: microtubuli only appear within a time- and space frame that integrates tubulin activation, (de)polymerization and diffusion rates. ${ }^{19}$ This concept has inspired the temporal programming of synthetic analogues, varying from 1D nanofibers to macroscale gels that form and fall apart as dictated by the kinetics of the underlying chemical reactions. ${ }^{20-26}$

Spatial programmability in self-organizing systems, however, is more challenging to establish, especially if one aims to translate interactions at the (supra)molecular level into spatiotemporally controlled 
phenomena at mesoscopic length scales. Typically, spatial control in synthetic systems involves external stimuli that contain spatial information, such as concentration gradients ${ }^{27}$, external fields ${ }^{28-30}$ or localized photo-control. ${ }^{31}$ Nature, however, demonstrates how spatial self-organization emerges from the bottomup: multiple dissipative assembly and diffusion steps together constitute complex positioning processes of, for example, the centrosome - involved in chromosome segregation during cell division. ${ }^{32}$ In synthetic systems, reaction-diffusion ${ }^{33}$ and convection ${ }^{34}$ have been identified as driving forces in the emergence of spatial patterns from initially homogeneous conditions. Although the complexity of these features is far from the complexity found in nature, these results exemplify that fluxes that maintain concentration gradients serve as a means to coordinate spatial differentiation - prerequisite to obtain spatial selforganization. We reasoned that fluxes which coordinate the positioning of their own driving forces enable a general design principle to program positioning routines in molecular systems. Here, we demonstrate how the coupling between self-assembly at the molecular scale and self-sustained fluxes resulting from the concentration gradients that evolve around the self-assembled features, enable autonomous positioning of dynamic assemblies in analogy to the centrosome.

\section{Marangoni flows lead to repulsive and attractive forces in a dissipative system}

The design of our system relies on the amphiphile tetra(ethylene glycol) monododecyl ether $\left(\mathrm{C}_{12} \mathrm{E}_{4} \mathrm{OH}\right)$, which undergoes molecular self-assembly and, at the same time, shows interesting surfactant release/depletion dynamics at air-water interfaces. Linear amphiphiles such as $\mathrm{C}_{12} \mathrm{E}_{4} \mathrm{OH}$ are known to form a laminar phase of closely packed bilayers in aqueous solutions. ${ }^{35-37}$ At the edge of concentrated amphiphile droplets, the spaces in between these bilayers take up more water; the resulting pressure forces the packed bilayers to buckle and filaments, consisting of packed cylinders of amphiphile bilayers, grow at the boundary of the amphiphile droplet (Fig. 1a). In literature, small angle X-ray scattering studies combined with freeze-fracture transmission electron microscopy on cross-sections have shown that these protrusions - also called "myelins" - consist of packed cylinders of amphiphile bilayers, separated by water with a spacing in the order of $10-100 \mathrm{~nm} .^{38,39}$ To initiate the assembly of $\mathrm{C}_{12} \mathrm{E}_{4} \mathrm{OH}$ into filaments, a $0.5 \mu \mathrm{L}$ source droplet of $40 \mathrm{v} / \mathrm{v} \% \mathrm{C}_{12} \mathrm{E}_{4} \mathrm{OH}$ in a water/ethanol mixture was deposited on the air-water interface of an aqueous sodium alginate solution (see Methods). Optical microscopy images reveal a blobbed surface of the droplet and after a few minutes multiple, approx. 10-50 $\mu \mathrm{m}$ thick filaments emerge, that grow in all directions and extend for several millimeters (Fig. 1b, Supplementary Movie 1). As the amphiphile has a density $<1 \mathrm{~g} \mathrm{~mL}^{-1}$, the filaments float at the air-water interface. Upon deposition of the droplet, the surface tension drops instantly from $72 \mathrm{mN} \mathrm{m}^{-1}$ (pure water) to $29 \mathrm{mN} \mathrm{m}^{-1}$ (Supplementary Fig. 1), indicating that individual amphiphile molecules are released from the source droplet. The surface concentration of these 
amphiphiles approaches saturation, as a surface tension of $27 \mathrm{mN} \mathrm{m}^{-1}$ has been reported in literature for $\mathrm{C}_{12} \mathrm{E}_{4} \mathrm{OH}$ beyond its critical micelle concentration. ${ }^{40}$

We observed that the initial decrease in surface tension reversed over time, when amphiphiles slowly dissolved into the underlying aqueous phase (Supplementary Fig. 1). This inspired us to introduce a drain droplet of a hydrophobic liquid that would deplete the amphipiles from the interface, thus setting up a permanent gradient in amphiphile distribution at the air-water interface. Hence, an out-of-equilibrium system with a stable Marangoni flow would be established. When the rate of amphiphile release from the source (1) is larger than depletion at the drain (2), the Marangoni flow pushes the source and drain droplets apart (Fig. 1a bottom). At the same time, the self-assembled amphiphile filaments are extruded from the droplet by the Marangoni flow towards the drain, and get absorbed by the drain droplet upon arrival (3, Fig. 1a bottom). As can be seen in Fig. 1c (Supplementary Movie 2), filament absorption pulls the drain, a droplet of oleic acid with sodium oleate, towards the source, which means a net force is exerted on the drain droplet during absorption. As we will show below, matching the rates of processes 1-3 results in dynamic self-positioning of the source and drain droplets. 

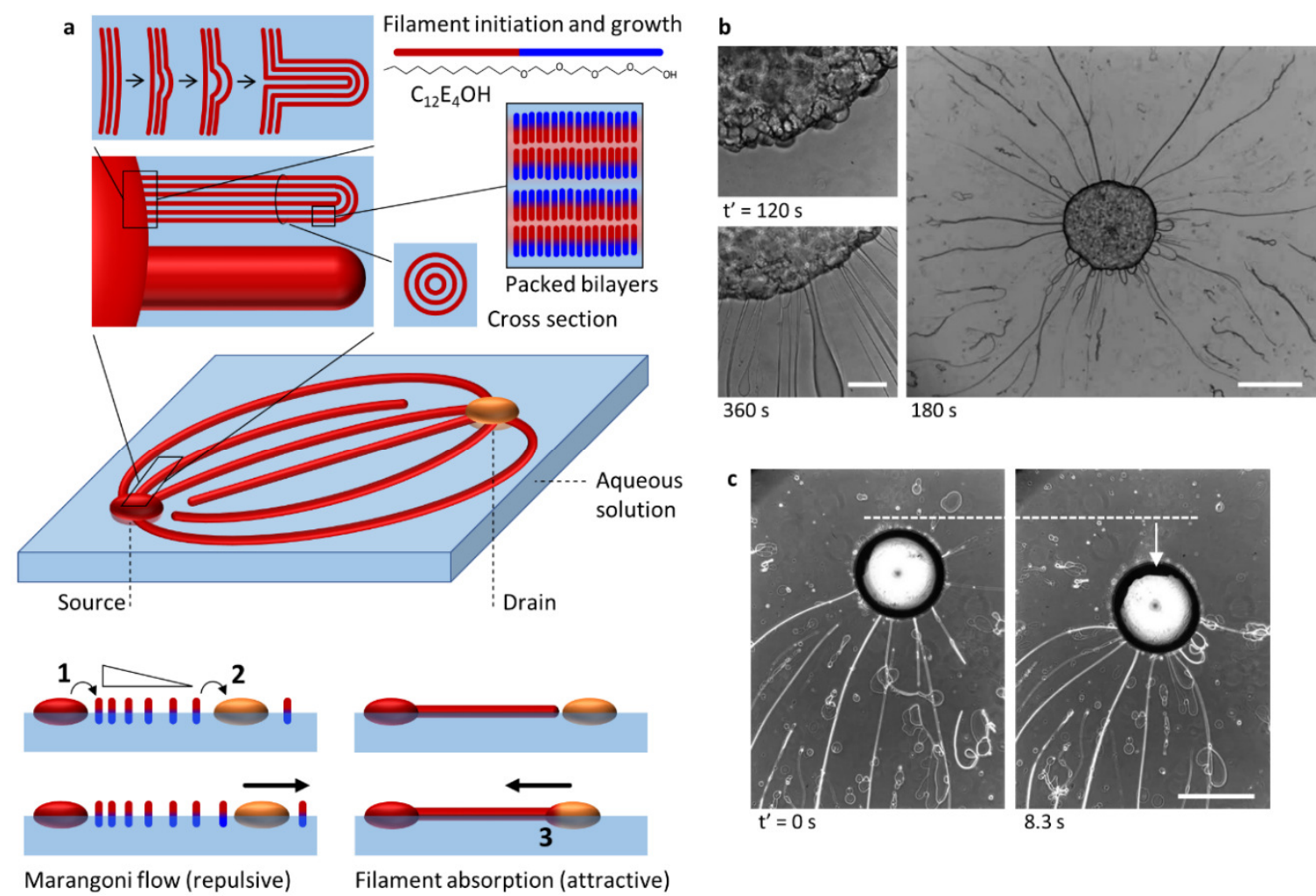

Marangoni flow (repulsive)

Filament absorption (attractive)

Fig. 1 I Marangoni flows and filaments direct positioning. a, In the source droplet (red sphere), $\mathrm{C}_{12} \mathrm{E}_{4} \mathrm{OH}$ forms closely packed bilayers. At the droplet boundary, the amphiphiles take up water (light blue), the packed bilayers buckle and form filaments consisting of bilayers, as schematically represented in the top left. Concomitantly, individual amphiphile molecules are released form the source to the air-water interface, and subsequently deplete at the drain (orange sphere), such that a Marangoni flow emerges that pushes the drain and source droplets apart (bottom left, 1, 2). The drain is however pulled back to the source upon absorption of the filaments (bottom right, 3). A dynamic organization emerges when the repulsive (Marangoni flow) and attractive forces (filament absorption) match. b, Optical microscopy images of filament extrusion from the amphiphile source droplet floating at an aqueous sodium alginate solution. The left scale bar represents $200 \mu \mathrm{m}$; the right scale bar $1 \mathrm{~mm}$. c, Upon filament absorption, the drain droplet is pulled towards the source - positioned in the bottom with respect to the area featured in the microscopy images. The left scale bar represents $1 \mathrm{~mm}$.

\section{Balancing the repulsive and attractive forces}

To assess how this Marangoni flow relies on both the rates of amphiphile release from the source droplet and depletion at the drain droplet, we developed a kinetic model that predicts the surface tension when both an amphiphile source droplet and a drain droplet are present at the air-water interface. In the model (schematically presented in Figure $2 \mathrm{a}$ and elaborated in the Methods Section), $A_{\mathrm{s}}$ equals the density 
of amphiphiles present in the source droplet and filaments, $\Gamma$ the density of amphiphiles present as surfactant at the air-water interface, $\theta$ the density of vacant sites at the air-water interface, $A_{\mathrm{d}}$ the density of amphiphiles in the drain and $A_{\mathrm{m}}$ the density of amphiphiles dissolved in the underlying aqueous phase, respectively - all densities (in $\mathrm{mol} \mathrm{cm}^{-2}$ ) are averaged over the whole area. The irreversible release of amphiphiles from source droplet and filaments to the air-water interface is described with rate constant $k_{1}$ (reaction 1 in Fig. 2a); the irreversible depletion at the drain with rate constant $k_{2}$ and rate equation $\mathrm{d} \Gamma / \mathrm{d} t=$ $-k_{2} \cdot\left(\Gamma-\Gamma_{\text {eq }}\right)$ (reaction 2); and the depletion from the air-water interface to the underlying aqueous phase with rate constant $k_{3}$ and equilibrium constant $K_{3}$ (reaction 3 ). The surface tension value is derived from the amphiphile density at the air-water interface via the Frumkin adsorption isotherm. ${ }^{40}$

Simulations provided us with deeper insight into how the different processes affect the selforganization. As shown in Figure 2b, the surface tension is predicted to drop from $72 \mathrm{mN} \mathrm{m}^{-1}$ to $28 \mathrm{mN}$ $\mathrm{m}^{-1}$ when the amphiphile source droplet is applied at $t=0 \mathrm{~s}$. Deposition of a drain droplet at $t=10 \mathrm{~min}$ switches $k_{2}=0$ (no drain) to $k_{2}>0$ (drain effective). When the amphiphile depletion at the drain outpaces the release of new amphiphiles from the source, that means: $k_{1} \cdot A_{\mathrm{s}} \cdot \theta<k_{2} \cdot\left(\Gamma-\Gamma_{\text {eq }}\right)$, a new steady state sets in at a higher surface tension value. This fast amphiphile depletion results in a strong Marangoni flow towards the drain (regime 1 in Fig. 2c), which was observed when oleic acid was deposited as a drain: the surface tension increased, the drain instantly attracted filaments and pulled the droplets together, leading to merging of source and drain within a few seconds (Fig. 2d, Supplementary Movie 3, Supplementary Fig. 2). These observations suggest that a stable self-organization of source and drain droplets requires a reduced uptake of amphiphiles at the drain, such that $k_{1} \cdot A_{\mathrm{s}} \cdot \theta>k_{2} \cdot\left(\Gamma-\Gamma_{\mathrm{eq}}\right)$ and the surface tension remains constant after deposition of the drain.

To reduce the amphiphile depletion rate (i.e. $\left.k_{2}\right)$, we included sodium oleate $(10 \mathrm{wt} \%)$ in the oleic acid drain. Upon deposition, the surface tension remained constant, indicating that the amphiphile release from the source was faster than the depletion at the drain, i.e. $k_{1} \cdot A_{\mathrm{s}} \cdot \theta>k_{2} \cdot\left(\Gamma-\Gamma_{\mathrm{eq}}\right)$ (Supplementary Fig. 2). As a result, the Marangoni flow pushed the drain away from the source, following regime 2 (Fig. 2e, Supplementary Movie 3). However, when the drain was deposited such that it hit a number of filaments originating from the source, the filaments tethered to the drain (regime 3). Upon absorption of these filaments, the drain was kept in position and the droplets maintained a center-to-center separation of 1-4 $\mathrm{mm}$ over the course of more than 1 hour, while filaments were slowly transferred from source to drain (Fig. 2 fg, Supplementary Fig. 3, Supplementary Movie 3). Together, these results demonstrate that if $k_{1} \cdot A_{\mathrm{s}} \cdot \theta>$ $k_{2} \cdot\left(\Gamma-\Gamma_{\mathrm{eq}}\right)$ and the filaments tether to the drain, the positioning of free-floating source and drain droplets is maintained by the balance between $i$ ) the Marangoni flow which pushes the drain away, and ii) the absorption of filaments, which causes the drain to be pulled along these filaments toward the source. 

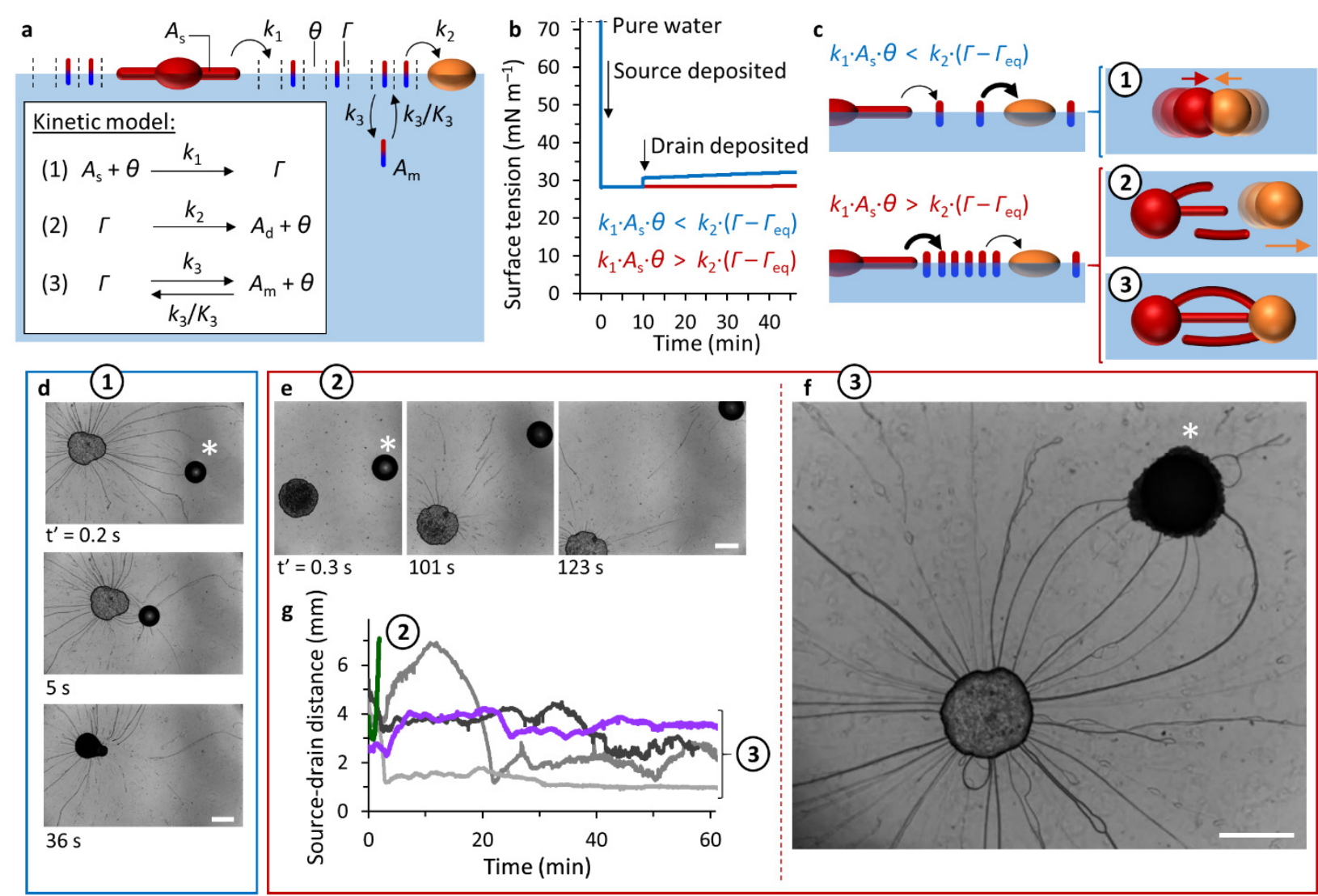

Fig. 2 | Droplet positioning relies on balanced rates of amphiphile release, depletion and filament absorption.

a, Kinetic model, describing 1) the release of amphiphiles from the source (red sphere) to the air-water interface, 2) depletion of amphiphiles at the drain (orange sphere), and 3) depletion of amphiphiles to the underlying aqueous phase. b, Simulations of surface tension kinetics upon deposition of the source and drain droplets. The surface tension increases only if the uptake of amphiphiles at the drain outpaces the release of amphiphiles from the source (blue curve). c, Three different regimes can be classified: d, In regime 1, the drain (asterisk) merged with the source droplet. e, In regime 2, the drain was slowly pushed away from the source. f, In regime 3, the filaments connected the source to the drain, such that it was kept in position. g, Time-dependent center-to-center source-drain distance: the green curve corresponds to the experiment shown in (e); the purple curve to the experiment shown in (f) and the grey curves to separate experiments performed under similar conditions (Supplementary Fig. 3). The scale bars represent $1 \mathrm{~mm}$. 


\section{Autonomous positioning of multi-droplet systems}

To demonstrate how the well-balanced coupling between self-assembly and gradient-driven molecular fluxes in regime 3 drives autonomous positioning, we established the positioning of a freefloating source droplet in a ring of fixed drain droplets - resulting in assemblies that self-organize in analogy to the centrosome. The drain droplets were positioned at the tips of six pins, hexagonally positioned on a $5.8 \mathrm{~mm}$ diameter ring placed in the aqueous solution. When the source droplet was deposited, it moved around and occasionally bounced into one of the drains. However, when long filaments started to grow, tethering of filaments to the drains aided in the positioning of the source droplet, which maintained its position within the hexagon over the course of more than 1 hour (Fig. 3a-d, Supplementary Movie 4, Supplementary Fig. 4). When no drain droplets were deposited at the pins, the source droplet moved out of the hexagon (Fig. 3cd, Supplementary Fig. 5). Together, these results imply that the Marangoni flow drags the filaments towards the drains. Potentially, this flow also drags the source altogether towards one of the drains. However, when tethering of filaments to all drains has been established, drag of the source towards one drain is inhibited by its connection to the other drains. The robustness of this self-positioning was exemplified by depositing a new source droplet in the hexagon. The filaments expelled the new droplet from the hexagon, allowing the original system to recover (Fig. 3e, Supplementary Movie 5). 


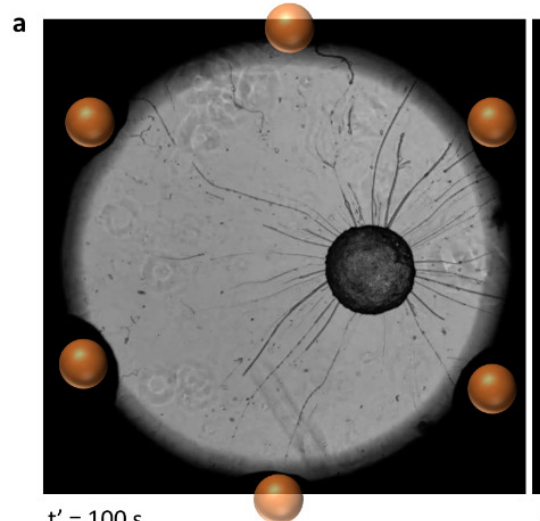

$t^{\prime}=100 s$

b
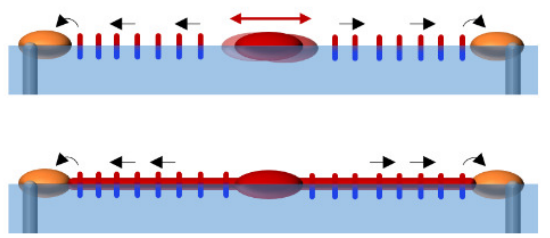

$55 \min$

c

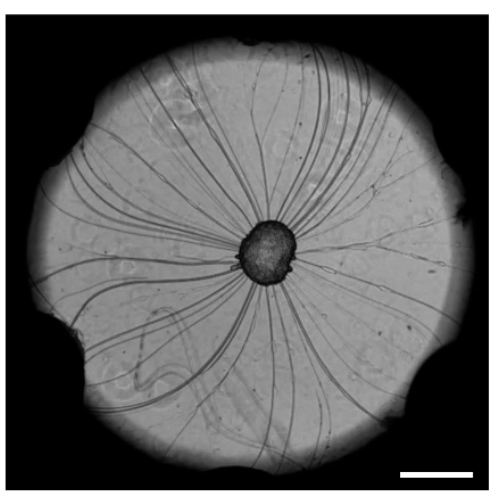

$73 \mathrm{~min}$
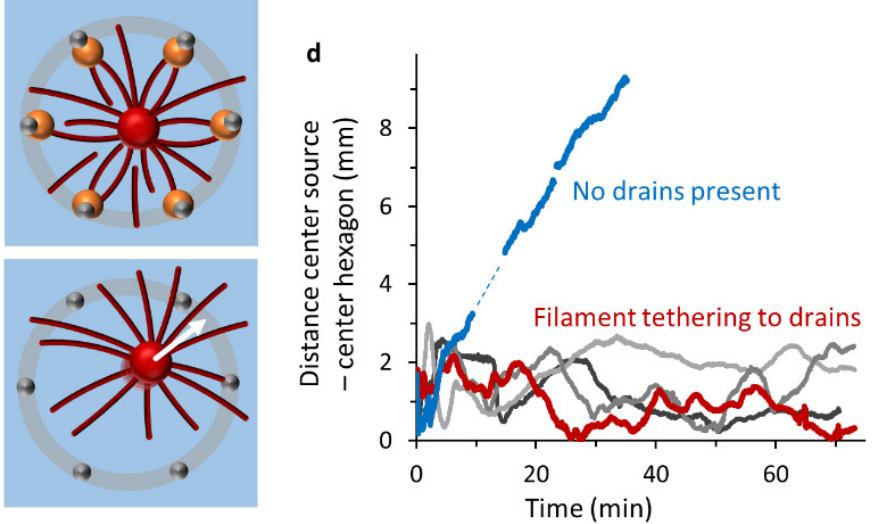

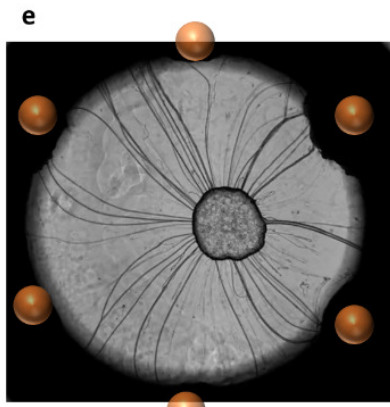

$t^{\prime}=-6 s$

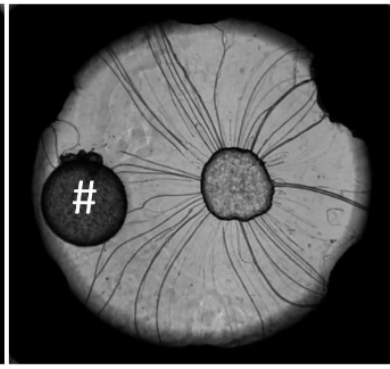

$\mathrm{t}^{\prime}=4 \mathrm{~s}$

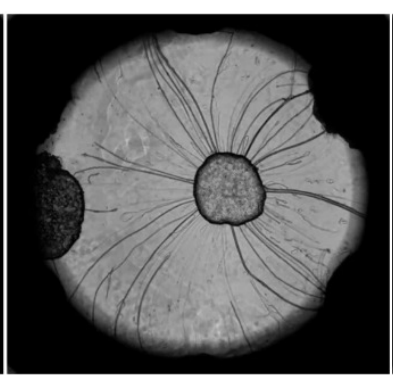

$38 \mathrm{~s}$

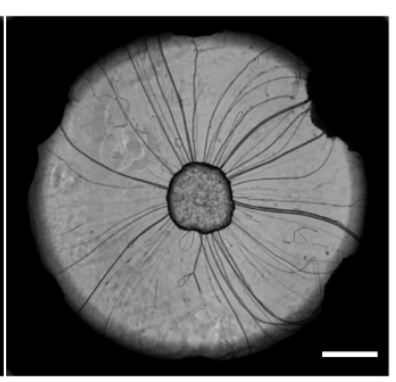

$188 \mathrm{~s}$

Fig. 3 I Autonomous positioning of dynamic assemblies in a ring of drain droplets. a, Optical microscopy recording of a free-floating source droplet self-positioning in between six drain droplets that were placed at the tips of hexagonally positioned pins - as indicated by the orange spheres in the left pane. b, Schematic representation of the process: The Marangoni flow towards the drains moves the source droplet around (top pane), and drags the filaments towards the drains (bottom pane). c, Upon tethering to the drains, the filaments stabilize the positioning of the source droplet (top pane), whereas in the absence of drain droplets (bottom pane) the source droplet moves out of the hexagon. d, Time-dependent distance between the source droplet center and the hexagon center. The red curve corresponds to the experiment shown in (a), the grey curves correspond to separate experiments performed under similar conditions (Supplementary Fig. 4). The blue curve corresponds to the experiment that was conducted without drain droplets (Supplementary Fig. 5) e, The filaments of the central source droplet expelled a new source droplet (indicated with \#) that was deposited in the hexagon at $t^{\prime}=0 \mathrm{~s}$. The scale bars represent $1 \mathrm{~mm}$. 
Finally, we studied how Marangoni flow and filament self-assembly coordinate complex positioning in situations where all elements are mobile, i.e. free-floating. When a drain droplet was deposited in between two source droplets of pure $\mathrm{C}_{12} \mathrm{E}_{4} \mathrm{OH}$, filaments from both sources were observed to grow toward the drain, and mediate the positioning of the droplets, as shown in Figure 4a (Supplementary Movie 6). Positioning follows the steps schematically outlined in Figure 4b-f: First, the opposing Marangoni flows from both sources repel the drain droplet from both sources (Fig. 4b). As the drain starts to "eat" the filaments that have arrived at the drain, it draws itself along these filaments to the respective source (Fig. 4c). As the drain gets closer to the source, more filaments tether to the drain: the capacity of the drain to absorb these filaments (mainly absorbing on one side of the drain) however is limited and hence, the growing filaments push the source away from the source (Fig. 4d). In the meantime, filaments that grow from the opposite source droplet arrive and tether to the backside of the drain (Fig. 4e). Their absorption causes the drain to be pulled back, such that it positions itself in between the source droplets (Fig. 4f). The resulting self-organization remained stable over $>5$ hours (Supplementary Fig. 6); NB the increased filament density was obtained by increasing the $\mathrm{C}_{12} \mathrm{E}_{4} \mathrm{OH}$ content to $100 \%$ in the source droplets.

Importantly, the positioning relies on the balance between repulsion and attraction. When larger amounts of sodium oleate were included in the oleic acid drain (15 wt\%, $20 \mathrm{wt} \%$, Supplementary Fig. 7), a smaller number of filaments tethered to the drain, indicating that sodium oleate suppresses the capacity of the drain to attract and absorb filaments. Furthermore, when two drain droplets (10 wt $\%$ sodium oleate in oleic acid) were deposited in between the sources, instead of one, the overall filament absorption capacity increased and the drains ended up in a position much closer to the first source - comparable to the situation in Fig. 4d. An increase in the amount of sodium oleate led to a positioning of the two drain droplets further away from the sources, corroborating that the reduced absorption capacity decreased the attractive forces (Supplementary Fig. 8). To assess the versatility of this positioning mechanism, we deposited a drain droplet off-centered to the source droplets. As shown in Figure 4g (Supplementary Movie 7 and Supplementary Fig. 9), even though the Marangoni flows initially repelled the drain away from both source droplets, tethering of the filaments drew the drain back such that it, ultimately, ended up in between both source droplets - in analogy to the mechanism outlined in Figure 4b-f. 

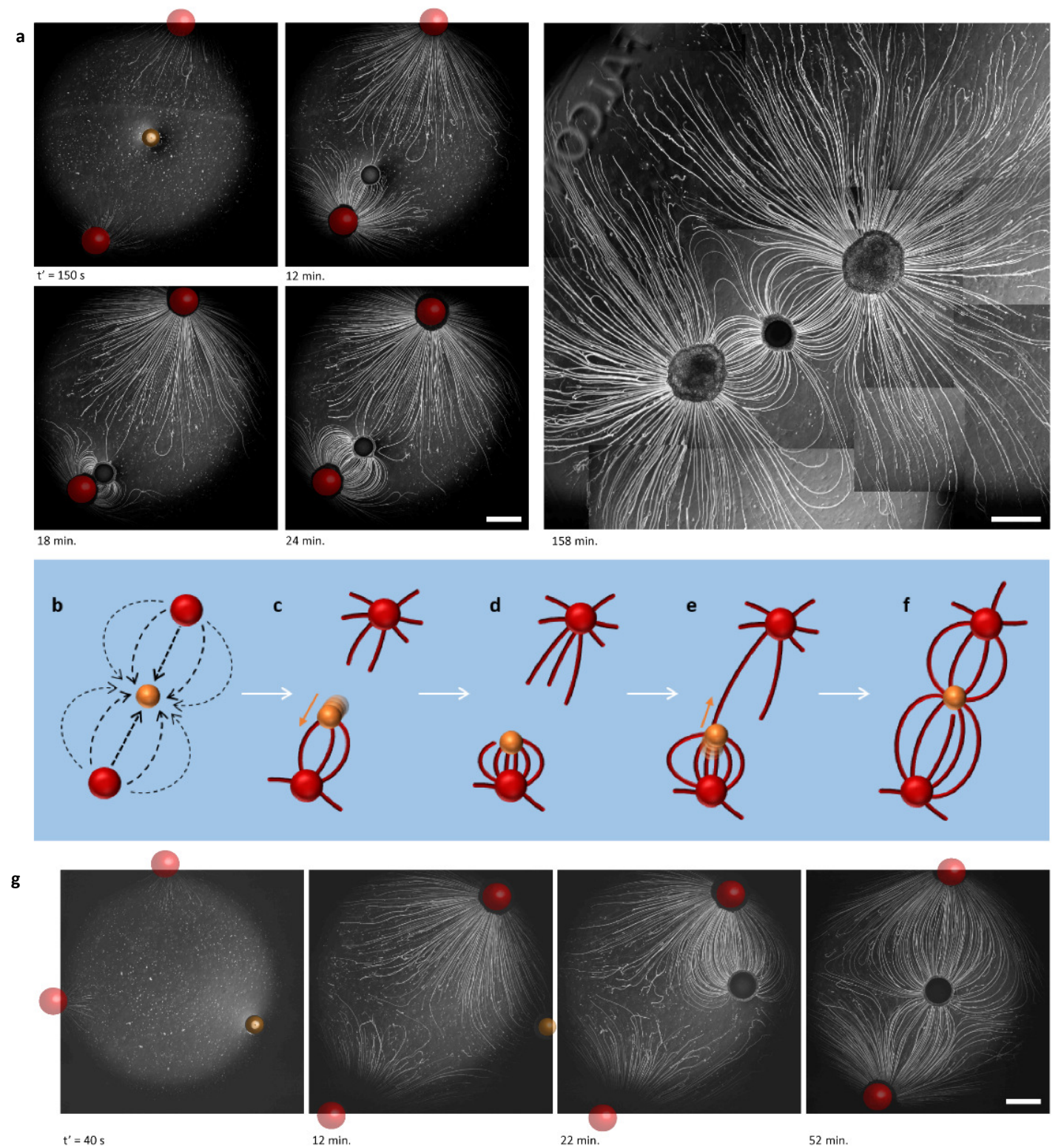

Fig. 4 I Capture and positioning of droplets by balancing Marangoni flow and absorption of filaments. a, Optical microscopy recording of a drain droplet (indicated by orange sphere) positioning in between two source droplets (indicated by red spheres). b-g, Proposed mechanism that mediates the positioning: $\mathbf{b}$, The Marangoni flows repel the drain (orange) from the two sources (red). c, The drain "eats" the filaments, and gets pulled along these filaments toward the source where these filaments originate from. $\mathbf{d}$, The large amount of filaments tethered to the drain, which has a limited overall filament absorption capacity, inhibits the drain to stay too close to the source. e, Filaments that originate from the other source arrive and tether to the backside of the drain. $\mathbf{f}$, Absorption of filaments from both directions mediates positioning of the drain in between both sources. $\mathbf{g}$, After deposition of the drain droplet off-centered to the two source droplets, absorption of filaments drew the drain towards the top source. Subsequently, upon tethering of filaments from the bottom source, the drain was positioned in between the two source droplets. The scale bar represents $2 \mathrm{~mm}$. 


\section{Conclusion}

We have demonstrated a system that displays mesoscale self-organization, driven by self-sustained out-of-equilibrium fluxes of amphiphiles that mediate the positioning of their origin. On the one hand, Marangoni flows push source and drain droplets apart when the rates of amphiphile release and depletion from the air-water interface match. At the same time, the amphiphile molecules self-assemble into filaments which grow along these flows and tether the drain to the source, such that pushing flows and tethering assemblies together keep the droplets in position and self-organization is sustained.

Concentration gradients offer a general design principle to provide spatial differentiation at mesoscopic length scales. We note that droplet-based systems - often in combination with dynamic nanostructures such as surfactants, nanoparticles or non-equilibrium assemblies - are gaining popularity as a platform to explore the emergence of complex, collective behavior in multi-component systems, with interest varying from origin-of-life contexts ${ }^{41,42}$ to multiphase reactors ${ }^{43}$, sensors ${ }^{44,45}$, reconfigurable objects $^{46,47}$, optics ${ }^{48}$ and maze solving. ${ }^{49,50}$ The concept of self-assembling, wire-like structures that are transferred amongst droplets and concomitantly aid in their positioning can become useful in scenarios where transfer of signals adds programmable coordination in self-organization behavior. Additional feedback mechanisms, e.g. by delivering chemicals that enhance or counteract a local depletion of surfactants, might enable autonomously operating systems that form and re-wire connections along 2D substrates - opening new possibilities in dynamic, smart materials.

\section{Acknowledgements}

We thank the Dutch Research Council (NWO, Veni grant no. 722.016.009 and START-UP grant no. 740.018.003) as well as the Ministry of Education, Culture and Science (Gravity program, 024.001.035) for financial support. We thank J. Aizenberg for stimulating discussions.

\section{Author Contributions}

P.A.K. conceived and supervised the project, W.T.S.H. contributed to the multi-droplet self-positioning concept, which was experimentally established by A.v.d.W. M.W., A.v.d.W., S.M.C.S. and P.A.K. performed the experiments. P.A.K. developed the model. P.A.K., W.T.S.H. and M.W. wrote the paper.

\section{Competing interests}

The authors declare no competing interests. 


\section{References}

1. Karsenti, E. Self-organization in cell biology: a brief history. Nat. Rev. Mol. Cell Biol. 9, 255-262 (2008).

2. Gelebart, A. H., Mulder, D. J., Varga, M., Konya, A., Vantomme, G., Meijer, E. W., Selinger, R. L. B \& Broer, D. J. Making waves in a photoactive polymer film. Nature 546, 632-636 (2017).

3. Orlova, T., Lancia, F., Loussert, C., Iamsaard, S., Katsonis, N. \& Brasselet, E. Revolving supramolecular chiral structures powered by light in nanomotor-doped liquid crystals. Nat. Nanotechnol. 13, 304-308 (2018).

4. Chen, J., Leung, F. K.-C., Stuart, M. C. A., Kajitani, T., Fukushima, T., Van der Giessen, E. \& Feringa, B. L. Artificial muscle-like function from hierarchical supramolecular assembly of photoresponsive molecular motors. Nature Chem. 10, 132-138 (2018)

5. Qian, X., Zhao, Y., Alsaid, Y., Wang, X., Hua, M., Galy, T., Gopalakrishna, H., Yang, Y., Cui, J., Liu, N., Marszewski, M., Pilon, L., Jiang, H. \& He, X. Artificial phototropism for omnidirectional tracking and harvesting of light. Nat. Nanotechnol. (2019) DOI: 10.1038/s41565-019-0562-3.

6. Huber, L., Suzuki, R., Krüger, T., Frey, E. \& Bausch, A.R. Emergence of coexisting ordered states in active matter systems. Science 361, 255-258 (2018).

7. Cangialosi, A., Yoon, C., Liu, J., Huang, Q., Guo, J., Nguyen, T. D., Gracias, D. H. \& Schulman, R. DNA sequence-directed shape change of photopatterned hydrogels via high-degree swelling. Science 357, 11261130 (2017).

8. Gibaud, T., Barry, E., Zakhary, M.J., Henglin, M., Ward, A., Yang, Y., Berciu, C., Oldenbourg, R., Hagan, M.F., Nicastro, D., Meyer, R.B. \& Dogic, Z. Reconfigurable self-assembly through chiral control of interfacial tension. Nature 481, 348-351 (2012).

9. Wei, W.-S., Xia, Yl, Ettinger, S., Yang, S. \& Yodh, A.G. Molecular heterogeneity drives reconfigurable nematic liquid-crystal drops. Nature 576, 433-436 (2019).

10. Freeman, R., Han, M., Álvarez, Z., Lewis, J.A., Wester, J.R., Stephanopoulos, N., McClendon, M.T., Lynsky, C., Godbe, J.M., Sangji, H., Luijten, E. \& Stupp, S.I. Reversible self-assembly of superstructured networks. Science 362, 808-813 (2018).

11. There's plenty of room at the top. Nat. Nanotechnol. 14, 729 (2019).

12. Leira-Iglesias, J., Tassoni, A., Adachi, T., Stich, M. \& Hermans, T.M. Oscillations, travelling fronts and patterns in a supramolecular system. Nat. Nanotechnol. 13, 1021-1027 (2018).

13. Ragazzon, G. \& Prins, L. J. Energy consumption in chemical fuel-driven self-assembly. Nat. Nanotechnol. 13, 882-889 (2018).

14. England, J.L. Dissipative adaptation in driven self-assembly. Nat. Nanotechnol. 10, 919-923 (2015).

15. Te Brinke, E., Groen, J., Herrmann, A., Heus, H.A., Rivas, G., Spruijt, E. \& Huck, W.T.S. Dissipative adaptation in driven self-assembly leading to self-dividing fibrils. Nat. Nanotechnol. 13, 849-855 (2018).

16. Grzybowski, B.A. \& Huck, W.T.S. The nanotechnology of life-inspired systems. Nat. Nanotechnol. 11, 585-592 (2016).

17. Mattia, E. \& Otto, S. Supramolecular systems chemistry. Nat. Nanotechnol. 10, 111-119 (2015).

18. Ashkenasy, G., Hermans, T.M., Otto, S. \& Taylor, A.F. Systems Chemistry. Chem. Soc. Rev. 46, $2543-$ 2554 (2017).

19. Kirschner, M. \& Mitchison, T. Beyond self-assembly: from microtubules to morphogenesis. Cell 45, 329342 (1986).

20. Boekhoven, J., Hendriksen, W.E., Koper, G.J.M., Eelkema, R. \& Van Esch, J.H. Transient assembly of active materials fueled by a chemical reaction. Science 349, 1075-1079 (2015).

21. Morrow, S.M., Colomer, I. \& Fletcher, S.P. A chemically fuelled self-replicator. Nat. Commun. 10, 1011 (2019).

22. Grötsch, R.K., Wanzke, C., Speckbacher, M., Angi, A., Rieger, B. \& Boekhoven, J. Pathway Dependence in the Fuel-Driven Dissipative Self-Assembly of Nanoparticles. J. Am. Chem. Soc. 141, 9872-9878 (2019).

23. Nakashima, K.K., Baaij, J.F. \& Spruijt, E. Reversible generation of coacervate droplets in an enzymatic network. Soft Matter 14, 361-367 (2018). 
24. Heuser, T., Weyandt, E. \& Walther, A. Biocatalytic Feedback-Driven Temporal Programming of SelfRegulating Peptide Hydrogels. Angew. Chem. Int. Ed. 54, 13258-13262 (2015).

25. Kumar, M., Ing, N.L., Narang, V., Wijerathne, N.K., Hochbaum, A.I. \& Ulijn, R.V. Amino-acid-encoded biocatalytic self-assembly enables the formation of transient conducting nanostructures. Nature Chem. 10, 696-703 (2018).

26. Mishra, A., Korlepara, D. B., Kumar, M., Jain, A., Jonnalagadda, N., Bejagam, K.K., Balasubramanian, S. \& George, S.J. Biomimetic temporal self-assembly via fuel-driven controlled supramolecular polymerization. Nat. Comm. 9, 1295 (2018).

27. Zadorin, A.S., Rondelez, Y., Gines, G., Dilhas, V., Urtel, G., Zambrano, A., Galas, J.-C. \& Estevez-Torres, A. Synthesis and materialization of a reaction-diffusion French flag pattern. Nature Chem. 9, 990-996 (2017).

28. Singh, G., Chan, H., Baskin, A., Gelman, E., Repnin, N., Král, P. \& Klajn, R. Self-assembly of magnetite nanocubes into helical superstructures. Science 345, 1149-1153 (2014).

29. Demirörs, A.F., Pillai, P.P., Kowalczyk, B. \& Grzybowski, B.A. Colloidal assembly directed by virtual magnetic moulds. Nature 503, 99-103 (2013).

30. Wang, W., Timonen, J.V.I., Carlson, A., Drotlef, D.-M., Zhang, C.T., Kolle, S., Grinthal, A., Wong, T.-S., Hatton, B., Kang, S.H., Kennedy, S., Chi, J., Blough, R.T., Sitti, M., Mahadevan, L. \& Aizenberg, J. Multifunctional ferrofluid-infused surfaces with reconfigurable multiscale topography. Nature 559, 77-82 (2018).

31. Ross, T. D., Lee, J. J., Qu, Z., Banks, R. A., Phillips, R. \& Thomson, M. Controlling organization and forces in active matter through optically defined boundaries. Nature 572, 224-229 (2019).

32. Laan, L., Pavin, N., Husson, J., Romet-Lemonne, G., Van Duijn, M., Preciado López, M., Vale, R.D., Jülicher, F., Reck-Peterson, S.L. \& Dogterom, M. Cortical Dynein Controls Microtubule Dynamics to Generate Pulling Forces that Position Microtubule Asters. Cell 148, 502-514 (2012).

33. J. Horváth, Szalai, I. \& De Kepper, P. An experimental design method leading to chemical Turing patterns. Science 324, 772-775 (2009).

34. Zhang, Y., Tsitkov, S. \& Hess, H. Complex dynamics in a two-enzyme reaction network with substrate competition. Nature Catalysis 1, 276-281 (2018).

35. Mitchell, D.J., Tiddy, G.J.T., Waring, L., Bostock, T. \& McDonald, M.P. Phase behaviour of polyoxyethylene surfactants with water. J. Chem. Soc., Faraday Trans. I 79, 975-1000 (1983).

36. Buchanan, M., Egelhaaf, S.U. \& Cates, M.E. Dynamics of interface instabilities in nonionic lamellar phases. Langmuir 16, 3718-3726 (2000).

37. Zou, L.-N. \& Nagel, S.R. Stability and growth of single myelin figures. Phys. Rev. Lett. 96138301 (2006).

38. Sakurai, I., Suzuki, T. \& Sakurai, S. Cross-sectional view of myelin figures. Biochim. et Biophys. Acta 985, $101-105$ (1989).

39. Chen, X. \& Tsujii, K. Synthetic myelin figures immobilized in polymer gels. Soft Matter 3, 852-856 (2007).

40. Hsu, C.-T., Shao, M.-J. \& Lin, S.-Y. Adsorption kinetics of C12E4 at the air-water interface: adsorption into a fresh interface. Langmuir 16, 3187 - 3194 (2000).

41. Rodríguez-Arco, L., Li, M. \& Mann, S. Phagocytosis-inspired behavior in synthetic protocell communities of compartmentalized colloidal objects. Nature Mater. 16, 857-863 (2017).

42. Parilla Gutierrez, J.M., Hinkley, T., Taylor, J.W., Yanev, K. \& Cronin, L. Evolution of oil droplets in a chemorobotic platform. Nature Commun. 5, 5571 (2014).

43. Yang, Z., Wei, J., Sobolev, Y.I. \& Grzybowski, B.A. Systems of mechanized and reactive droplets powered by multi-responsive surfactants. Nature 553, 313-318 (2018).

44. Zhang, Q., Savagatrup, S., Kaplonek, P., Seeberger, P.H. \& Swager, T.M. Janus emulsions for the detection of bacteria. ACS Cent. Sci. 3, 309-313 (2017).

45. Cira, N.J., Benusiglio, A. \& Prakash, M. Vapour-mediated sensing and motility in two-component droplets. Nature 519, 446 - 450 (2015).

46. Liu, X., Kent, N., Ceballos, A., Streubel, R., Jiang, Y., Chai, Y., Kim, P.Y., Forth, J., Hellman, F., Shi, S., Wang, D., Helms, B.A., Ashby, P.D., Fischer, P. \& Russell, T.P. Reconfigurable ferromagnetic liquid droplets. Science 365, 264-257 (2019). 
47. Denkov, N., Tcholakova, S., Lesov, I., Cholakova, D. \& Smoukov, S.K. Self-shaping of oil droplets via the formation of intermediate rotator phases upon cooling. Nature 528, 392-395 (2015).

48. Goodling, A.E., Nagelberg, S., Kaehr, B., Meredith, C.H., Cheon, S.I. Saunders, A.P., Kolle, M. \& Zarzar, L.D. Colouration by total internal reflection and interference at microscale concave interfaces. Nature $\mathbf{5 6 6}$, 523-527 (2019).

49. Lagzi, I., Soh, S., Wesson, P.J., Browne, K.P. \& Grzybowski, B.A. Maze solving by chemotactic droplets. J. Am. Chem. Soc. 132, 1198-1199 (2010).

50. Jin, C., Krüger, C. \& Maass, C.C. Chemotaxis and autochemotaxis of self-propelling droplet swimmers. Proc. Natl. Acad. Sci USA 114, 5089-5094 (2017). 


\section{Methods}

Materials. Tetra(ethylene glycol) monododecyl ether $(\geq 98.0 \%)$ was purchased from Sigma-Aldrich and Santa Cruz Biotechnology Inc. (Dallas, TX). Sodium alginate and (+)-sodium L-ascorbate ( $\geq 99.0 \%)$ were purchased from Sigma-Aldrich, oleic acid from Fisher Chemical ( $\geq 70.0 \%)$ and Fluorochem (95\%), ethanol (absolute for analysis) from Merck and sodium oleate (95\%) from ABCR GmbH (Karlsruhe, Germany). All materials were used as received.

Source and drain solutions. The amphiphile source solution was prepared by mixing $40 \mathrm{v} / \mathrm{v} \%$ tetra(ethylene glycol) monododecyl ether $\mathrm{C}_{12} \mathrm{E}_{4} \mathrm{OH}, 15 \mathrm{v} / \mathrm{v} \%$ ethanol and $45 \mathrm{v} / \mathrm{v} \%$ water (unless stated otherwise). The drain solutions of oleic acid with sodium oleate were prepared upon dissolving sodium oleate in oleic acid upon sonication. The sodium alginate solution was prepared by dissolving sodium alginate in water $(6.25 \mathrm{mg} / \mathrm{mL})$. For the experiments that involved a drain droplet, also (+)-sodium Lascorbate $(3.4 \mathrm{mg} / \mathrm{mL}, 17 \mathrm{mM})$ was included in the sodium alginate solution.

Study self-organization with microscopy. Optical microscopy images and movies were acquired with an Olympus IX71 dark field inverted microscope equipped with a Phantom Vision camera, and an Olympus IX73 dark field inverted microscope equipped with a Point Grey Grasshopper3 camera. A 2x objective was used, unless stated otherwise. The frame rate was chosen appropriately; movies over the time course of approx. 1 hour were acquired at a frame rate of $1 \mathrm{fps}$. The composed microscopy image in Fig. 4a was assembled manually. To follow the positioning of the free-floating source and drain droplets over time (Fig. 2g and Fig. 3d), we analyzed the movie frames using Matlab (R2017a) and assessed the position of the circular droplets using the imfindcircles function.

To initiate the growth of the filaments, the amphiphile solution was deposited with a Gilson pipette at the interface of the sodium alginate solution in a polystyrene petridish (lid of a Falcon $35 \mathrm{~mm}$ dish, diameter $38 \mathrm{~mm}$, height $4.5 \mathrm{~mm}$, used as received). In order to prevent the droplet from moving towards the solution meniscus at the edge of the petridish, the petridish was filled completely with sodium alginate solution, such that a convex air-water interface was formed $(5.5 \mathrm{~mL}$, solution height $4.85 \mathrm{~mm})$. Prior to the deposition of the amphiphile droplet, the surface tension of the sodium alginate solution was decreased by adding a trace amount of amphiphile solution to the interface. All experiments were performed at room temperature, as a minimum temperature of approx. $20{ }^{\circ} \mathrm{C}$ has been reported in literature for the laminar phase of $\mathrm{C}_{12} \mathrm{E}_{4} \mathrm{OH}$, which is required to form the filaments (ref. 35). The formation of the laminar phase of $\mathrm{C}_{12} \mathrm{E}_{4} \mathrm{OH}$ in our experiments was verified by the opaque appearance, to the naked eye, of the $\mathrm{C}_{12} \mathrm{E}_{4} \mathrm{OH}$ droplet after deposition at the sodium alginate solution. 
In the experiment shown in Fig. $1 b, 0.5 \mu \mathrm{L}$ of the amphiphile solution was deposited. The images shown in the left were acquired at a larger magnification (10x objective); to keep the amphiphile droplet in position while acquiring those images, the droplet $(1.0 \mu \mathrm{L})$ was positioned at a metal pin (folded paperclip, diameter $0.75 \mathrm{~mm}$ ) that was inserted in the sodium alginate solution. In the experiment shown in Fig. $1 \mathrm{c}, 0.5 \mu \mathrm{L}$ of the amphiphile solution as well as $0.5 \mu \mathrm{L}$ of the drain solution (10 wt\% sodium oleate in oleic acid) was deposited at the sodium alginate solution.

In the experiment shown in Fig. $2 \mathrm{~d}, 0.5 \mu \mathrm{L}$ of the amphihile solution was deposited, and $0.5 \mu \mathrm{L}$ oleic acid. In Figs. $2 \mathrm{e}$ and $2 \mathrm{f}, 0.5 \mu \mathrm{L}$ of the amphihile solution was deposited, and $0.5 \mu \mathrm{L}$ of the drain solution (10 wt $\%$ sodium oleate in oleic acid).

In Fig. 3, a 3D printed hexagon (see Supplementary Fig. 4, 3D printed via fused filament technique using polylactic acid, inner ring diameter $5.8 \mathrm{~mm}$, pin height $5.2 \mathrm{~mm}$, pin thickness $1.8 \mathrm{~mm}$ ) was placed in the petri dish. The sodium alginate solution was applied such that the hexagon pins were touching the air-water interface and the air-water interface formed a concave meniscus at the hexagon pins. Then, the surface tension of the sodium alginate solution was decreased by touching the interface with a pipette tip with amphiphile solution; the drain droplets $(1.0 \mu \mathrm{L}, 10 \mathrm{wt} \%$ sodium oleate in oleic acid) were deposited at the tips of the pins, and the amphiphile solution $(1.0 \mu \mathrm{L})$ was deposited in the center of the hexagon.

In the experiments shown in Fig. 4, first two $1.0 \mu \mathrm{L}$ droplets of pure amphiphile were deposited simultaneously at the sodium alginate solution, using a Gilson 8-channel multipipette. After 25 seconds, $1.0 \mu \mathrm{L}$ of the drain solution (10 wt\% sodium oleate in oleic acid) was deposited at approximately equal distance to both source droplets (in the center of both source droplets for Fig. 4a; off-centered to both source droplets in Fig. 4g). The optical microscopy images were acquired at 1.25x magnification.

Surface tension experiments. The surface tension kinetics (Supplementary Figs. 1 and 2) were acquired using a KSV Instruments LTD surface tensiometer that measures the force exerted by the air-water meniscus on a platinum Wilhelmy plate $(19.62 \mathrm{~mm}$ x $10 \mathrm{~mm})$ inserted into pure MQ water. For the experiment shown in Supplementary Fig. 1, first a Teflon Langmuir-Blodgett (LB) trough (7.5 cm x 32.5 $\mathrm{cm}$ ) was cleaned upon subsequent rinsing with water, ethanol and water, and drying using pressurized nitrogen. Next, the through was filled with MQ water, and the barriers of the LB-setup were closed slowly in order to compress a monolayer of potential contaminants occupying the air-water interface. Next, the upper layer of the water in between the barriers was removed upon suction, new water was added from behind the barriers to substitute the removed water, and the barriers were opened again. The Wilhelmy plate was cleaned with water and ethanol, flamed until red hot, and inserted into the water, such that the water wetted the plate and formed a meniscus. The surface tension was set at $72 \mathrm{mN} \mathrm{m}^{-1}$ for pure water, 
and after two minutes, the amphiphile solution was deposited at the air-water interface, ca. $10 \mathrm{~cm}$ away from the Wilhelmy plate. For the experiments in Supplementary Fig. 2, where the surface tension kinetics were followed upon successively depositing an amphiphile source droplet and a drain droplet, polystyrene petridishes (Falcon, diameter $35 \mathrm{~mm}$, height $9.5 \mathrm{~mm}$, used as received) were filled with $5 \mathrm{~mL}$ MQ water (solution height $5.2 \mathrm{~mm}$ ). The droplets were deposited such that they moved towards the meniscus at the edge of the petri dish, and did not get into contact with each other.

Kinetic model to simulate surface tension kinetics. The kinetic model that predicts the surface tension during the self-organization process involves the irreversible release of the amphiphile from the source droplet to the air-water interface with rate constant $k_{1}$ (reaction 1 in Fig. 2a), the irreversible depletion of amphiphile at the drain droplet with rate constant $k_{2}$ (reaction 2) and the reversible depletion of amphiphile from the air-water interface to the bulk phase of the aqueous solution with rate constant $k_{3}$ and equilibrium constant $K_{3}$ (reaction 3). We simulated the density of amphiphile present as individual surfactant at the airwater interface $(\Gamma)$, based on which we computed the surface tension value (vide infra). Furthermore, $A_{\mathrm{s}}$ equals the density of amphiphiles present in both the source droplet as well as the filaments, $\theta$ the density of vacant sites at the interface, and $A_{\mathrm{m}}$ the density of amphiphiles in the bulk phase of the aqueous solution. All density parameters are in $\mathrm{mol} \mathrm{cm}^{-2}$, and averaged over the whole area: our model does not involve spatial specification.

Based on the reaction equations presented in Fig. 2a, we obtain the following rate equations:

(2) $\frac{d \theta}{d t}=-k_{1} \cdot A_{s} \cdot \theta+k_{3} \cdot \Gamma-k_{3}^{\prime} \cdot A_{m} \cdot \theta+k_{2}\left(\Gamma-\Gamma_{e q}\right)$ vacant sites at interface

(3) $\frac{d \Gamma}{d t}=k_{1} \cdot A_{s} \cdot \theta-k_{3} \cdot \Gamma+k_{3}^{\prime} \cdot A_{m} \cdot \theta-k_{2}\left(\Gamma-\Gamma_{e q}\right)$ amphiphile at air-water interface

$$
\frac{d A_{m}}{d t}=k_{3} \cdot \Gamma-k_{3}^{\prime} \cdot A_{m} \cdot \theta
$$

amphiphile in aqueous medium

To compute the surface tension $\gamma$ (in $\mathrm{mN} \mathrm{m}^{-1}$ ) based on $\Gamma$, we used the Frumkin isotherm, as reported by Hsu et al. (ref. 40):

(5) $\gamma=\gamma_{0}+10^{7} \cdot \Gamma_{\infty} R T\left(\ln \left(1-\frac{\Gamma}{\Gamma_{\infty}}\right)-\frac{K}{2}\left(\frac{\Gamma}{\Gamma_{\infty}}\right)^{2}\right)$ 
Here, $\gamma_{0}$ represents the surface tension of the clean air-water interface $\left(72 \mathrm{mN} \mathrm{m}^{-1}\right.$ for pure water); $R$ the gas constant, $T$ the temperature $(T=293 \mathrm{~K}), K$ the adsorption cooperativity factor $(K=1.875)$ and $\Gamma_{\infty}$ the maximum surface concentration $\left(\Gamma_{\infty}=4.663 \cdot 10^{-10} \mathrm{~mol} \mathrm{~cm}^{-2}\right){ }^{40}$

To simulate the surface tension kinetics, we defined the starting values for $A_{\mathrm{s}}, \theta, \Gamma$ and $A_{\mathrm{m}}$. Initially, all amphiphiles are present in the source droplet, implying that $A_{\mathrm{s}}(t=0)$ can be derived from the volume of the amphiphile source droplet and the area of the air-water interface, and $\Gamma(t=0)=0$ and $A_{\mathrm{m}}(t=0)=0$. The density of vacant positions available at the air-water interface was derived from literature data on the equilibrium density of amphiphiles present at the air-water interface just beyond the critical micelle concentration (cmc). Based on the Frumkin isotherm, and data reported by Hsu et al (ref. 40), we derived $\theta(t=0)=4.44 \cdot 10^{-10} \mathrm{~mol} \mathrm{~cm}{ }^{-2}$.

The simulations shown in Fig. $2 \mathrm{~b}$ were performed with $k_{1}=9.6 \cdot 10^{7} \mathrm{~cm}^{2} \mathrm{~mol}^{-1} \mathrm{~s}^{-1} ; k_{3}=1.2 \cdot 10^{-2} \mathrm{~s}^{-1}$; $K_{3}=1.1 \cdot 10^{-9} \mathrm{~mol} \mathrm{~cm}^{-2} ; \Gamma_{\mathrm{eq}}=4.33 \cdot 10^{-10} \mathrm{~mol} \mathrm{~cm}{ }^{-2}$ and $A_{\mathrm{s}}(t=0)=5.42 \cdot 10^{-8} \mathrm{~mol} \mathrm{~cm}^{-2}$. For the regime where $k_{1} \cdot A_{\mathrm{s}} \cdot \theta<k_{2} \cdot\left(\Gamma-\Gamma_{\mathrm{eq}}\right)$, the simulation was performed with $k_{3}=5 \mathrm{~s}^{-1}$; for the regime where $k_{1} \cdot A_{\mathrm{s}} \cdot \theta>$ $k_{2} \cdot\left(\Gamma-\Gamma_{\text {eq }}\right)$, the simulation was performed with $k_{2}=0.05 \mathrm{~s}^{-1}$. At $t=10 \mathrm{~min}$, i.e. right before the transition from $k_{2}=0$ to $k_{2}>0, A_{\mathrm{s}}=5.06 \cdot 10^{-8} \mathrm{~mol} \mathrm{~cm} \mathrm{~cm}^{-2} ; \theta=1.09 \cdot 10^{-12} \mathrm{~mol} \mathrm{~cm}{ }^{-2}$ and $\Gamma=4.43 \cdot 10^{-10} \mathrm{~mol} \mathrm{~cm}^{-2}$. Hence, $k_{1} \cdot A_{\mathrm{s}} \cdot \theta=5.29 \cdot 10^{-12} \mathrm{~mol} \mathrm{~cm} \mathrm{~cm}^{-2}$. With $k_{2}=5 \mathrm{~s}^{-1}, k_{2} \cdot\left(\Gamma-\Gamma_{\mathrm{eq}}\right)=4.79 \cdot 10^{-11} \mathrm{~mol} \mathrm{~cm} \mathrm{c}^{-2} \mathrm{~s}^{-1}$ and with $k_{2}=0.05 \mathrm{~s}^{-1}$, $k_{2} \cdot\left(\Gamma-\Gamma_{\mathrm{eq}}\right)=4.79 \cdot 10^{-13} \mathrm{~mol} \mathrm{~cm}^{-2} \mathrm{~s}^{-1}$. These values imply that for both regimes, the given inequalities are met. 